\title{
Finite-Level Quantized Consensus of Multi-agent Networked Systems
}

\author{
Olubusola O. Nuga - Kamoli A. \\ Amusa - Simeon Matthew - Ayorinde J. \\ Olanipekun • Adebayo Abayomi-Alli
}

Received: date / Accepted: date

\begin{abstract}
This paper is concerned with the analysis of consensus multi-agent networked system. Adopted in the analysis is the finite-level logarithmic quantizer, for the transmission of the networked-agents state. Two protocols are utilised in the analysed multi-agent networked system: the consensus protocol, which is determined from the outputs and states of a set of encoder-decoder pair that is employed in the system, and convergence rate protocol that is precisely characterized via the use of a dynamic scaling factor. With information exchange among neighbouring agents, the asymptotic consensus can be reached. The proof of protocols is based on proper selection of parameters of the logarithmic quantizer chosen for the connected network. As a demonstration of the validity of the protocols, a four-agent networked system is used. It is shown that an undirected network exchange of information via a communication channel that is equipped with a set of encoder and decoder can lead to attainment of estimates of neighbour state protocol for the networked system. Furthermore, desired asymptotic convergence can be reached through appropriate choice of parameters of the logarithmic quantizer.
\end{abstract}

Keywords Quantization · Finite-level · Logarithmic · Consensus · networked

\section{Introduction}

The problem of consensus of Multi-Agent Systems (MASs) has attracted the attention of many researchers involved in control systems and allied scientific fields, such as unmanned vehicles, automated systems, mini-satellites communication networks, etc. $[27,14]$. Previous efforts into the study of consensus

O. O. Nuga

Federal University of Agriculture Abeokuta, Electrical and Electronic Engineering, Nigeria E-mail: nugaoo@funaab.edu.ng

A. Abayomi-Alli

Federal University of Agriculture Abeokuta, Computer Science, Nigeria 
protocols include $[5,1,26,12]$ to mention a few. In recent times, extensive research in the area of consensus control of MASs is ongoing $[20,25,21,6,17$, $13]$.

Lately, quantized consensus problems of dynamic agents have been a subject of interest because of their theoretical and practical importance in advanced technologies such as hybrid systems, discrete-network, digital control and information constraint. Some of the studies considered the consensus problems of MASs under diverse conditions, such as dynamics of discrete- and continuous- (with or without) time-delays, static or dynamic communication networks, among others $[27,14,28,24,21,6]$.

The coordination of vehicle problems is considered in [6] where inter-vehicle communication is used to realise the vehicle action management. The method of decentralized information exchange between vehicles is proposed for vehicle management. The investigation carried out in [28] on the consensus problem of dynamic agents proved the convergence analysis and protocol performance. In [27], consensus problems of MASs with common dynamics of linear node as well as fixed directed protocols are considered. Based on the given relative periodic information, a fresh set of distributed protocols emerged. The protocols guarantee consensus so long the rate of information transfer and the stabilizability of each agent are above the threshold. A distributed observertype-based controller is presented in [14] as the outcome of the investigation of the consensus problem of MASs with time-invariant network topology involving general dynamics of the linear node. The controller is established based on the relative measured outputs. While [24] on the other hand, studied the consensus problem in MASs, wherein the information about the states of all agents tend toward a time-dependent reference position, where only a few of the agents is assumed to have access to the reference state. Presented by [19] is a proposal of two linear and time-varying gain consensus algorithms in an effort of addressing the consensus problem involving a single integrator MASs. Preset time for the required state in directed and undirected communication networks is achieved. Systems involved in those aforementioned studies are continuous-time-based. Hence, the need to investigate discrete-time-based systems. The discrete-time systems require extensive digital controllers for the MASs to function as Networked Control Systems (NCSs). The relationship between communication and control systems, in reality, considering the system's performance, considers communication constraints arising in NCSs.

Effects of non-uniform time-delays and dynamical topologies on consensus problem of MASs are studied by [18] where a linear consensus protocol that establishes local control schemes for the second-order discrete-time agents is suggested. In the communication process, quantized information affects the process output, which limits the channel bandwidth. The ignored quantized errors in many control designs, and analytical methods are a result of its complexity $[7,4]$. In $[10,8]$, the authors considered some quantized feedback design problems for a linear system aiming at stabilizing the given system. Likewise, a particular performance with the coarsest quantization density was achieved, minimizing the quantization levels to attain a specified control desire. Mo- 
tivated by the average consensus problem on a network of digital links, [4] proposed sets of algorithms established on pairwise "gossip" communication. A new design method is introduced in [2], this depends on how sensitive the quantizer changes while the system evolves, interconnected with the given system resulting in a hybrid system. The joint significance of dynamics of agents, the topology of the network and communication on discrete-time linear MASs is investigated by [29]; through the introduction of few necessary and sufficient conditions. The consensus control of an average undirected network comprising several discrete-time first-order agents that are communication constraints is considered in [15]. The problem is addressed through a design of a distributed protocol that establishes dynamic encoding and decoding of agents states. In a related effort, [31] approached the problem of distributed consensus of discrete-time agents through the introduction of agents' states broadcast via infinite logarithmic quantization. In addition, a controller based on the states and outputs of the channel is projected.

This paper studies the quantized logarithmic consensus of MASs with finite bits rate. Each agent has a real-valued state at which the exchange of important information with neighbours can be realized. For protocol specification, consideration is given to error compensation defined by the bit rate of MASs, control system gain and scaling factor. The main focus is on finite-level logarithmic quantized networked of a discrete-time system based on the enhanced procedure of the states and outputs of a set of encoders and decoders. However, for appropriately selected control parameters with a one-bit quantizer, consensus can be reached. Also examined is the connection between the number of quantization levels and the convergence rate; faster convergence requires more bits. Hence, the asymptotic rate of convergence of quantized systems is dependent on the number of bits, the consensusability of the system and the number of MASs involved in the network.

The rest of the paper is structured as follows: while section 2 presents an overview of the graph theory and quantization, section 3 highlights the problem under study. The convergence analysis of finite-level quantized consensus is contained in section 4 . This paves the way to section 5 , where numerical results are presented to corroborate the validity of the protocols formed using the four-agent system in the illustration. Section 6 concludes the paper.

\section{Notation and preliminaries}

Network topology of a system of $N$-linked agents can be modelled either as a directed or undirected graph denoted by $\mathcal{G}=(\mathcal{V}, \mathcal{E})$. The communication network modelled through a graph $\mathcal{G}$, consisting of a vertex/node set $(\mathcal{G}) \mathcal{V}=$ $1,2, \ldots N$ also, an edge set $\mathcal{E}(\mathcal{G}) \subset \mathcal{V} \times \mathcal{V}$. Each vertex represents an agent, the edge $(i, j)$ shows that the agents $i$ and $j$ share the information about their states, if $i, j \in \mathcal{V}$ and $i, j \in \mathcal{E}$ then $i$ and $j$ are adjacent, that is, agents connected by an edge referred to as neighbours, the relation, is represented by $i j$ and assumes that $i i$ always holds. 
Various matrices are by nature connected with graphs, such as the adjacency matrix, the diagonal matrix, and the Laplacian matrix. Given a graph $\mathcal{G}$, the formed matrix contains information about the structured graph.

The graph adjacency matrix $\mathcal{G}=(\mathcal{V}, \mathcal{E})$, described by $\mathcal{A}$, is the rows and columns of the integer matrix that is indexed by vertices of $\mathcal{G}$, such that $a_{i j}=1, a_{i i}=0$ if $(j, i) \in \mathcal{E}$ or else 0 . The graph Laplacian matrix $\mathcal{G}=(\mathcal{V}, \mathcal{E})$, symbolized by $\mathcal{L}$, is the rows and columns of the matrix is indexed by the vertices of $G$, such that $\mathcal{L}_{i i}=\sum_{j \neq i} a_{i j}, \mathcal{L}_{i j}=-a_{i j}$ for $i \neq j$. This matrix is closely related to adjacency matrix $\mathcal{A}$ of $\mathcal{G}$. Given $\mathcal{D}$ to be diagonal matrix of a graph $\mathcal{G}=(\mathcal{V}, \mathcal{E})$, whose rows and columns are indexed by vertices of $\mathcal{G}$, with diagonal entries $\mathcal{D}_{i i}=d_{i}$, defined as: $\mathcal{D}_{i j}=\operatorname{deg}\left(v_{i}\right)$, alternatively, or else 0 , which is equivalent to: $\mathcal{L}=\mathcal{D}-\mathcal{A}$ The adjacency and Laplacian matrices are positive semidefinite by definition; furthermore, for directed graphs, they show symmetric structure. The eigenvalues of the Laplacian matrix $\mathcal{L}$ in the increasing order represented by $0=\lambda_{1}(\mathcal{L}) \leq \lambda_{2}(\mathcal{L}) \leq \ldots \lambda_{N}(\mathcal{L})$, and the spectral radius given as $\lambda_{N}(\mathcal{L})$ of $(\mathcal{L}) . A \otimes B$ denotes the Kronecker product or tensor product of $A$ and $B$. Resultant matrices satisfy the following properties: 1) $(A \otimes B)(C \otimes D)=A C \otimes B D, 2)(A \otimes B)^{T}=A^{T} \otimes B^{T}$ also, 3) $(A \otimes B)^{*}=A^{*} \otimes B^{*}$

Lemma 1 [25], if a Laplacian matrix $\mathcal{L} \in R^{n \times n}, l_{i j} \leq 0, l_{i j} \geq 0, \forall i \neq j$, and $\sum_{j=1}^{N} l_{i j}=0$ for each $j$, then $\mathcal{L}$ has at least one zero eigenvalues, in which, all the nonzero eigenvalues occupy the open left half plane. Besides, $\mathcal{L}$ would have precisely one zero eigenvalues, given the spanning tree in $\mathcal{L}$ is a directed graph.

Linear quantization has some advantages but is not the ideal choice for many applications, while logarithmic quantization is intended for a high dynamic range. Each channel has a set of decoder and encoder where all agent receives the signalled information from; then the estimated neighboring state is obtained by a decoding algorithm. The logarithmic quantizer is adopted such that the state of each agent is quantized. $q_{l}(x)$ given as $[11,22,16,30]$ :

$$
q_{l}(x)= \begin{cases}u_{i}, & \frac{u_{i}}{1+\nu}<x \leq \frac{u_{i}}{1-\nu} \\ 0, & x=0 \\ -q_{l}(-x), & x<0\end{cases}
$$

where $\nu=\frac{1-\iota}{1+\iota}$ determines the quantization density of $q_{l}(x) \cdot q_{l}(x)$ is the set such that $\mathcal{U}=\left\{ \pm u_{i}: u_{i}=\iota_{i} u_{0}, i= \pm 1, \pm 2, \ldots\right\} \cup\left\{ \pm u_{0}\right\} \cup\{0\} \quad 0<\iota<1, u_{0}>$ 0 the quantizer is easily bounded by a sector bound such as: $\left|q_{l}(x)-x\right| \leq \Delta|x|$ where $\Delta \in[-\nu, \nu]$. Hence, the lower the value of $\nu$, the higher the precision the quantized logarithmic signal.

The logarithmic quantizer is illustrated in Figure 1 which shows the sector bound. The primary objective is to use the quantizer information to reach the stability of the overall closed-loop system. The Lyapunov function of the quadratic stabilization problem $V(x)=x^{T} P x, P^{T}=P>0$ is deployed to determine the system feedback stability. Text with citations [?] and [?]. 


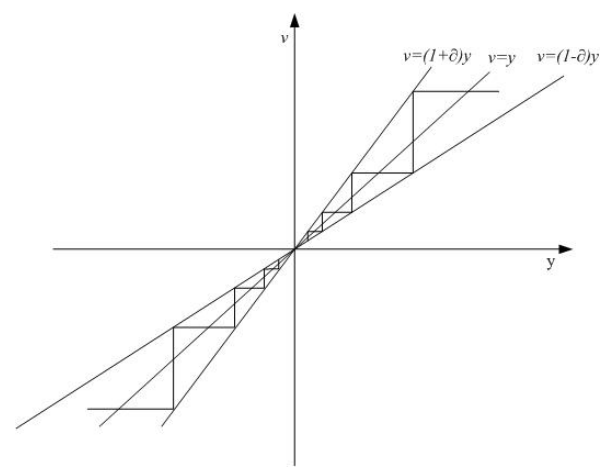

Fig. 1 Logarithmic Quantizer

\section{Problem Statement}

Considering a class of network of MASs comprising $(N)$ agents, with the linear dynamic system, the $i t h$ agents can be expressed as

$$
x_{i}(k+1)=A x_{i}(k)+B u_{i}(k) \quad i=1,2, \ldots, N
$$

where $x_{i}(k) \in \mathbf{R}^{N}$, denote the real-valued state of the agents, and $u_{i}(k) \in \mathbf{R}^{P}$ is the control input. Matrices $A \in \mathbf{R}^{N \times N}$ is assumed unstable, and $B \in$ $\mathbf{R}^{N \times M}$, is non-varying, while both are stabilisable matrices.

For a controllable system $A, B$ the feedback control law should be designed as $u_{i}=-K x_{i}$ for each subsystem, such that $A-B K$ is stable. The possible control law for controller is given

$$
u_{i}=K \sum_{j=1}^{n} l_{i j} x_{j}(k)
$$

where $K$ is the controller gain, and the Laplacian matrix $\mathcal{L}$ elements $l_{i j}$ in the same notion

$$
u_{i}(k)=-K \sum_{j=1}^{n} a_{i j}\left(x_{i}(k)-x_{j}(k)\right) .
$$

The controller uses all accessible information around the neighbourhood from the analysis in (2).

\subsection{Consensus Control Feedback System}

Using the given control law, the closed loop system gives a state feedback algorithm described by (2) and (4)

$$
x_{i}(k+1)=A x_{i}(k)-B K \sum_{j=1}^{n} a_{i j}\left(x_{i}(k)-x_{j}(k)\right)
$$


The quantized logarithmic in (1) examines the number of infinite quantized level. Based on the limitation in (1) it feasible to vary the quantizer inputoutput signal to achieve asymptotic stability via introduction of logarithmic quantizer finite-level. Introducing an $2 Q+1$-level with a quantization density, $q_{l}(x)$ is given as $[4,11]: \mathcal{U}=\left\{ \pm u_{i}, i=0,1,2, \ldots, Q\right\} u_{0}>0$. The corresponding $Q($.$) is given as$

$$
q_{l}(x)= \begin{cases}u_{i}, & \frac{u_{i}}{1+\nu}<x \leq \frac{u_{i}}{1-\nu}, \quad 0 \leq i \leq Q-1 \\ u_{Q-1}, & 0 \leq x \leq \frac{u_{Q}-1}{1-\nu}, \\ 1, & x>\frac{1}{1+\nu}, \\ -q_{l}(-x), & x<0,\end{cases}
$$

The digital channels transmit signal data, which is assumed to be reliable, i.e. noiseless, due to the requirement in (4) of the communication channel in which the actual state valued of each agent can not be accessed by the neighboring agents at each time interval. Each channel has a pair of encodersdecoders. When each agent accepts the signal data from the neighbors, then the estimated neighbor state is obtained by a decoding algorithm. The logarithmic quantizer is adopted to determine the state of each of the agents. The encoder $\phi_{i}$ of $i$ th agent sent to its neighbours is given as

$$
\begin{aligned}
x_{i}(0) & =0, \\
x_{i}(k+1) & =A \hat{x}_{i j}(k)+\frac{q_{l} \times g(k+1)\left(x_{i}(k+1)-A \hat{x}_{i j}(k)\right)}{g(k+1)}
\end{aligned}
$$

provided $x_{i}(k+1)$ indicates the inner state and $\frac{q_{l} \times\left(x_{i}(k+1)-A \hat{x}_{i j}(k)\right)}{g(k+1)}$ is the output of $\phi_{i}$. The $q_{l}($.$) is the finite-level logarithmic quantizer, and g(k)>0$ is the scaling factor (gain). Initializing $g(0)$ to be positive integer, the constants $\gamma_{1}, \gamma_{2} \in(0,1)$ define $g(k+1)$ whenever $t \geq 0$ in which [10]

$$
g(k+1)= \begin{cases}g(k) \gamma_{1}, & \left|q_{l}(g(k) x(k))\right|=1 \\ g(k) / \gamma_{2}, & \left|q_{l}(g(k) x(k))\right|<1 \\ g(k), & \text { otherwise }\end{cases}
$$

The $q_{l}():. \mathbf{R} \rightarrow \mathcal{U}$, is considered the symmetric quantizer.

Remark 1 The scaling factor influences the encoder $\phi_{i}$ and affects the magnitude of the predicted error, such that it is less than the state itself, which is expressed by fewer bits. If consensus is reached asymptotically, hence, the prediction error $x_{i}(k+1)-\hat{x}_{i} \rightarrow 0$ as $t \rightarrow \infty$.

Remark 2 Here $\gamma_{1}$ and $\gamma_{2}$ (scaling parameters) keeps the scaled input $g(k) x(k)$ within the quantization range. The scaling parameters $\gamma_{1}$ and $\gamma_{2}$ either scale down or scale up $g(k+1)$ while $g(k)$ is zoomed-out, or zoomed-in. Note stability is determined by the scaling parameter. 
For every communication channel $(j, i) \in \varepsilon$, the $i$ th agent accepts information from the neighbor $j$ using the outputs of decoder $\psi_{i j}$ to update the state estimates of $x_{i}(k)$ as follows [31]

$$
\begin{aligned}
\hat{x}_{i j}(0) & =0 \\
\hat{x}_{i j}(k+1) & =A \hat{x}_{i j}(k)+\frac{q_{l} \times g(k+1)\left(x_{i}(k+1)-A \hat{x}_{i j}(k)\right)}{g(k+1)}
\end{aligned}
$$

where $\hat{x}_{i j}(k)$ is the output of $\psi_{i j}(k)$.

Note that the output of the quantizer determines the signal flow among the agents. Hence, status of an agent is a function of its quantized state and those of its neigbhours.

Meanwhile the choice of $g(k)$ does not influence stabilizability. $Q()=$. $[q(.), \ldots, q(.)]^{T}$, is compatible with the input. From (9) the controller (4) is written as:

$$
u_{i}(t)=-K \sum_{j=1}^{N} a_{i j}\left(\hat{x}_{i j}(t)-x_{j}(t)\right)
$$

The $x_{j}(t)$ correction error is replaced by $\hat{x}_{j}(t)$, with initial state $\hat{x}_{i}(0)=0$. The product quantizer $q_{l}($.$) is defined by q_{l}()=.\left[q_{l}(.), \ldots, q_{l}(.)\right]^{T}$, which is compatible with the input. From (9) the controller (4) is written as:

$$
u_{i}(t)=-K \sum_{j=1}^{N} a_{i j}\left(\hat{x}_{i j}(t)-\hat{x}_{j}(t)\right)
$$

Denote $x_{i}(t)=\left[x_{1}^{T}, \ldots, x_{N}^{T}\right]^{T}$ the total state of the system, $\hat{x}_{i}(t)=\left[\hat{x}_{1}^{T}, \ldots, \hat{x}_{N}^{T}\right]^{T}$ error state, $e_{i}(t)=x_{i}(t)-\hat{x}_{i}(t)$ the quantized state error, $\delta_{i}(t)=x_{i}(t)-1_{N} \otimes$ $\bar{x}_{i}(t)$.

Using (7) and (9),

$$
\begin{aligned}
\hat{x}_{i j}(t)=x_{j}(t), \quad t=0,1,2, \ldots \quad i, j \in N, \quad i=1,2, \ldots, N \\
u_{i}(t)=-K \sum_{j=1}^{N} a_{i j}\left[x_{i}(t)-x_{j}(t)-\left(x_{i}(t)-\hat{x}_{i j}(t)\right)+\left(x_{j}(t)-x_{i}(t)\right)\right] \\
=-K \sum_{j=1}^{N} a_{i j}\left[x_{i}(t)-x_{j}(t)\right]+K \sum_{j=1}^{N} a_{i j}\left[x_{i}(t)-\hat{x}_{i j}(t)\right] \\
\quad-K \sum_{j=1}^{N} a_{i j}\left[x_{j}(t)-\hat{x}_{j i}(t)\right]
\end{aligned}
$$

In protocol (11) the input control of the $i$ th agent has three terms. The first term, $-K \sum_{j=1}^{N} a_{i j}\left[x_{i}(t)-x_{j}(t)\right]$, that plays the primary role represent the control input of protocol (4), second term $K \sum_{j=1}^{N} a_{i j}\left[x_{i}(t)-\hat{x}_{i}(t)\right]$ represent sum of the estimated errors for $x_{i}(t)$ by neighbors, while the last term 
$K \sum_{j=1}^{N} a_{i j}\left[x_{j}(t)-\hat{x}_{j}(t)\right]$ is the sum of estimated errors for the neighbor states $x_{j}(t)$. With protocol (6), (9) and (11) into protocol (2) the system becomes:

$$
\begin{aligned}
& x_{i}(t+1)=\left(I_{N} \otimes A-L \otimes B K\right) x_{i}(t)+(L \otimes B K) e_{i}(t) \\
& \hat{x}_{i}(t+1)=\frac{g(t+1) \times q_{l}\left(x_{i}(t+1)-\left(I_{N} \otimes A\right) \hat{x}_{i}(t)\right)}{g(t+1)}+\left(I_{N} \otimes A\right) \hat{x}_{i}(t)
\end{aligned}
$$

where $q_{l}\left(\left[x_{1}, \ldots, x_{N}\right]^{T}\right)=\left[q\left(x_{1}\right), \ldots, q\left(x_{N}\right)\right]^{T}$. rewritten as:

$$
\begin{aligned}
& x_{i}(t+1)=P x_{i}(t)+(\mathcal{L} \otimes B K) e_{i}(t) \\
& \hat{x}_{i}(t+1)=\frac{g(t+1) \times q_{l}\left(x_{i}(t+1)-\left(I_{N} \otimes A\right) \hat{x}_{i}(t)\right)}{g(t+1)}+\left(I_{N} \otimes A\right) \hat{x}_{i}(t)
\end{aligned}
$$

where $P \equiv\left(I_{N} \otimes A-\mathcal{L} \otimes B K\right) x_{i}(t)=\left(P_{i j}\right) \in \mathbf{R}^{N \times N}$ is the updated matrix, $q_{l}\left(\left[x_{1}, \ldots, x_{N}\right]^{T}\right)=\left[q_{l}\left(x_{1}\right), \ldots, q_{l}\left(x_{N}\right)\right]^{T}$ and $I_{N}$ is the identity matrix.

\section{Cnvergence analysis of finite-level quantized consensus}

The consensusability with the state feedback is subject to finite-level logarithmic quantized communication data rate of the protocol (14). This analysis can be carried out by considering the following in reaching consensus: choice of the finite-level quantizer parameters for the system; the number of bits needed for each agent to broadcast within their neighborhood.

With the following assumptions [15]: $\mathbf{A} \mathbf{1} \mathcal{G}$ is connected, $\mathbf{A} 2 \max _{1 \leq i \leq N}\left\|x_{i}(0)\right\| \leq$ $C_{x}, \max _{1 \leq i \leq N}\left\|\delta_{i}(0)\right\| \leq C_{\delta}$ such that $C_{x}$ and $C_{\delta}$ are noted nonnegative constant.

Lemma 2 Suppose assumption A1) holds then, given any control gain $t \in$ $\mathbf{R}^{1 \times N}$ defined as: $\left(A-\lambda_{i} B K\right)<1 \quad \forall i \in 2, \ldots, N \tau<K \lambda_{N}, \rho_{\tau}<1$,

$$
\rho_{\tau}=\max _{2 \leq i \leq N}\left|A-B K \lambda_{i}\right|
$$

Proof For any graph, $\lambda_{1}=0$, since $\mathcal{L} 1_{N}=0$. Therefore

$$
\left(A-\lambda_{i} B K\right)<1, \quad \rho_{\tau}<1
$$

$\lambda_{i}(\mathcal{L})$ and $\lambda_{i}(\overline{\mathcal{L}})$ have vector 1 and 0 as an eigenvector and eigenvalue, respectively. hence,

$$
=\left(A-\lambda_{i} B K\right)<\rho_{\tau}, \quad=\left(A-\lambda_{i} B K\right)<\max _{2 \leq i \leq N}\left|A-B K \lambda_{i}\right|
$$

Lemma 3 Assume $\mathbf{A} 1$ - A2 hold, for any $\gamma \in\left(\rho_{\tau}, 1\right)$ and $\tau \in\left(0, K \lambda_{N}\right]$, the following holds,

$$
M(\tau, \gamma)=\frac{\lambda_{N}^{2} N\|B K\|_{\infty}^{2} \nu}{\left(\gamma-\rho_{\tau}\right)}+\left(\|A\|_{\infty}+2 d_{\max }\|B K\|_{\infty}\right) \nu
$$


while for any given $\nu \in(0,1)$. Let $K$ be selected such that $K_{1} \in\left(0, \frac{1}{1+\nu}\right]$, and take the scaling factor $g(k+1)=g(0) \gamma_{1}$ or $g(k+1)=g(0) / \gamma_{2}$. Let $g(0)$ satisfies

$$
g(0) \geq \max \left\{\frac{\|A\|_{\infty} C_{x}}{K_{1}}, \frac{2\left(\gamma-\rho_{\tau}\right)\left(C_{\delta} \nu+\lambda_{N} C_{x}\|B K\|_{\infty}\right)}{\lambda_{N}\|B K\|_{\infty}}\right\}
$$

choosing the appropriate variables $\nu$ and $\gamma$ of logarithmic quantizer, discretetime consensus of the MASs (2), under quantizer information (6), (9) and the controller (11) consensus is said to be reached provided

$$
\lim _{t \rightarrow \infty} x_{i}(t)=\sum_{j=1}^{N} \lambda_{j} x_{j}(0), \quad i=1,2, \ldots, N
$$

Furthermore, the rate asymptotic convergence is bounded by $\gamma$, such that $r_{\text {asym }} \leq \gamma$.

Theorem 1 [15,23] Given a undirected graph $\mathcal{G}$, assume that assumption $\mathbf{A 1}$ - A2 holds, for any $\gamma \in\left(\rho_{\tau}, 1\right)$. Let,

$$
\begin{aligned}
& =\lim _{t \rightarrow \infty} \gamma^{-t}\|\delta(t)\|_{2} \\
& =\frac{g(0) \sqrt{N} \lambda_{N}\|B K\|_{\infty}}{\left(\gamma-\rho_{\tau}\right)} \nu
\end{aligned}
$$

Proof From (9)

$$
\hat{x}_{i}(t+1)=\frac{q_{l} \times g(t+1)\left(x_{i}(t+1)-A \hat{x}_{i}(t)\right)}{g(t+1)}+A \hat{x}_{i}(t)
$$

The estimated error $e(t)=x(t)-\hat{x}(t)$, by substituting (10) into (2)

$$
x_{i}(t+1)=A x_{i}(t)-B K \sum_{j=1}^{N} a_{i j}\left(x_{i}(t)-x_{j}(t)\right)+B K \sum_{j=1}^{N} a_{i j}\left(e_{i}(t)-e_{j}(t)\right)
$$

which is expressible as

$$
x_{i}(t+1)=P x_{i}(t)+(\mathcal{L} \otimes B K) e_{i}(t)
$$

where $L \mathbf{1}_{N}=\mathbf{0}$ and

$$
\begin{aligned}
& x_{i}(t+1)-\left(I_{N} \otimes A\right) \hat{x}(t) \\
& =\left(I_{N} \otimes A+\mathcal{L} \otimes B K\right) e_{i}(t)-(\mathcal{L} \otimes B K) \delta_{i}(t)
\end{aligned}
$$


the estimation error is specified as

$$
\begin{aligned}
\delta_{i}(t+1)= & P \delta_{i}(t)+(\mathcal{L} \otimes B K) e_{i}(t) \\
e_{i}(t+1)= & \left(I_{N} \otimes A+\mathcal{L} \otimes B K\right) e_{i}(t)-(\mathcal{L} \otimes B K) \delta_{i}(t) \\
& -\frac{1}{g(t+1)}\left(q_{l} \times g(t+1)\left(I_{N} \otimes A\right.\right. \\
& \left.+\mathcal{L} \otimes B K-\mathcal{L} \otimes B K) e_{i}(t)-(\mathcal{L} \otimes B K) \delta_{i}(t)\right)
\end{aligned}
$$

Represent $\omega(t)=\delta(t) / g(t+1), z(t)=e(t) / g(t+1)$ then $(24)$ and $(25)$ becomes

$$
\begin{aligned}
\gamma \omega(t+1)= & P \omega(t)+(\mathcal{L} \otimes B K) z(t) \\
\gamma z_{i}(t+1)= & \left(I_{N} \otimes A+\mathcal{L} \otimes B K\right) z_{i}(t)-(\mathcal{L} \otimes B K) \omega_{i}(t) \\
& -\frac{1}{g(t+1)}\left(q_{l} \times g(t+1)\left(I_{N} \otimes A+\mathcal{L} \otimes B K\right) z_{i}(t)-(\mathcal{L} \otimes B k) \omega_{i}(t)\right) \\
\omega_{i}(t+1) & =\gamma^{-1} P \omega_{i}(t)+\gamma^{-1}(\mathcal{L} \otimes B K) z_{i}(t) \\
z(t+1) & =\gamma^{-1} \beta
\end{aligned}
$$

where $\beta(t)$ is specified as $\beta(t)=\hat{e}(t)-q(\hat{e}(t))$ while $\hat{e}(t)=\left(I_{N} \otimes A+\mathcal{L} \otimes\right.$ $B K) z(t)-(\mathcal{L} \otimes B K) \omega(t)$ which can be noted as

$$
\begin{aligned}
\omega_{i}(t+1)= & \gamma^{-1} P \omega_{i}(t)+\gamma^{-1}(\mathcal{L} \otimes B K) z_{i}(t) \\
z_{i}(t+1)= & \gamma^{-1}\left(I_{N} \otimes A+\mathcal{L} \otimes B K\right) z_{i}(t) \\
& -\gamma^{-1}(\mathcal{L} \otimes B K) \omega_{i}(t) \\
& -\frac{1}{\gamma g(t+1)}\left(q_{l} \times g(t+1)\left(I_{N} \otimes A\right.\right. \\
& \left.+\mathcal{L} \otimes B K) z_{i}(t)-(\mathcal{L} \otimes B K) \omega_{i}(t)\right)
\end{aligned}
$$

Subsequently, the $2 Q+1$-level logarithmic quantizer in (6), $\left(I_{N} \otimes A+\mathcal{L} \otimes\right.$ $B K) z(t)-(\mathcal{L} \otimes B K) \omega(t)$ would be limited by choosing an appropriate value, $\gamma \in\left(\rho_{\tau}, 1\right)$ and $u_{0}$. From $(9) \hat{x}=0$ and $\mathcal{L} \mathbf{1}_{\mathbf{N}}=0$ therefore,

$$
\begin{aligned}
& \left\|\left(I_{N} \otimes A+\mathcal{L} \otimes B K\right) z_{i}(0)-(\mathcal{L} \otimes B K) \omega_{i}(0)\right\|_{\infty} \\
& =\|\left(I_{N} \otimes A+\mathcal{L} \otimes B K\right) x_{i}(0)-(\mathcal{L} \otimes B K)\left(x_{i}(0)\right. \\
& \quad-1_{N} \otimes \bar{x}_{i}(0)\left\|_{\infty}=\right\|\left(I_{N} \otimes A\right) x_{i}(0) \|_{\infty} \\
& \leq\|A\|_{\infty}\left\|x_{i}(0)\right\|_{\infty} \leq \rho_{i} u_{0}
\end{aligned}
$$

For some bounded initial state when $t=0$ the quantized level $\rho_{i} u_{0}$ of the logarithmic quantizer (6) is bounded. Also for some specified positive integer $t \geq 0$ suppose the input (6) is bounded. With reference to $\left|q_{l}(x)-x\right| \leq \Delta|x|$, the quantized level $u_{i} \geq u_{j}$ exists, then in view of (29) this implies:

$$
\|z(i)\|_{\infty} \leq \nu u_{j}=\frac{1-\iota}{1+\iota} u_{j}
$$


For a symmetric Laplacian matrix $\mathcal{L}$, the unitary matrix $\Phi=\left[1 / \sqrt{N}, \phi_{2} \ldots, \phi_{N}\right]$ defined by $\phi_{i}^{T} \mathcal{L}=\lambda_{i} \phi_{i}^{T}, i=2, \ldots, N$. Suppose $\tilde{\omega}(t)=\left(\Phi^{-1} \otimes I_{N}\right) \omega(t)=$ $\left(\Phi^{T} \otimes I_{N}\right) \omega(t)$ and $\tilde{\omega}(t)=\left[\tilde{\omega}_{1}^{T}(k), \tilde{\omega}_{2}^{T}(t)\right]^{T}$, where $\tilde{\omega}_{1}(t)=0$, can easily be verify. Denote $z(t)=\left(\Phi^{T} \mathcal{L} \otimes B K\right) z(t)=\left[\tilde{z}_{1}^{T}(t), \tilde{z}_{2}^{T}(t)\right]^{T}$. From $(26)$ :

$$
\begin{aligned}
\tilde{\omega}_{2}(t+1)= & \operatorname{diag}\left(\frac{A-\lambda_{2} B K}{\gamma}, \ldots, \frac{A-\lambda_{N} B K}{\gamma}\right) \gamma \tilde{\omega}_{2}(t)+\frac{1}{\gamma} \tilde{z}_{2}(t) \\
= & \left(\frac{\rho_{\tau}}{\gamma}\right)^{t+1}\left\|\tilde{\omega}_{2}(0)\right\|+\gamma^{-1}\left\|\Phi^{T} \mathcal{L}_{g} \otimes B K\right\|\left(\frac{\rho_{\tau}}{\gamma}\right)^{t} \| \tilde{z}_{2}(0 \|) \\
& +\gamma^{-1} \sum_{i=0}^{t}\left(\frac{\rho_{\tau}}{\gamma}\right)^{i}\left\|\Phi^{T} \mathcal{L}_{g} \otimes B K\right\|\left\|\tilde{z}_{2}(t-i)\right\|
\end{aligned}
$$

By estimating and regrouping the right-hand-side of (33) separately, recall for the first term, $\Phi$ is unitary matrix, $\left\|\tilde{\omega}_{2}(0)\right\|=\|\tilde{\omega}(0)\|=\|\omega(0)\| \leq$ $\sqrt{N}\|\delta(0)\|_{\infty}$, for any $N$ dimensional vector $\omega$, then:

$$
\left\|\left(\frac{\rho_{\tau}}{\gamma}\right)^{t+1} \tilde{\omega}_{2}(0)\right\| \leq\left(\frac{\rho_{\tau}}{\gamma}\right)^{t+1}\left\|\tilde{\omega}_{2}(0)\right\| \leq \frac{\sqrt{N}\|\delta(0)\|_{\infty}}{g(0)}\left(\frac{\rho_{\tau}}{\gamma}\right)^{t}
$$

While for the second term (33) using the assumption A2) $\gamma \in\left(\rho_{\tau}, 1\right)$ and $\|\mathcal{L}\|=\lambda_{N}$ is given as:

$$
\left\|\gamma^{-1} \Phi^{T} \mathcal{L} \otimes B K\left(\frac{\rho_{\tau}}{\gamma}\right)^{t} \tilde{z}_{2}(0)\right\| \leq \frac{\sqrt{N}\|B K\| C_{x} \lambda_{N}}{g(0)}\left(\frac{\rho_{\tau}}{\gamma}\right)^{t}
$$

Likewise, the upper limit of the last term in (33), by $\|z(i)\|_{\infty} \leq \nu u_{j}=$ $\frac{1-\iota}{1+\iota} u_{j}$ given as

$$
\left\|\sum_{i=0}^{t}\left(\frac{\rho_{\tau}}{\gamma}\right)^{i}\right\| \leq \sum_{i=0}^{t}\left\|\left(\frac{\rho_{\tau}}{\gamma}\right)\right\|^{i}=\frac{1-\left(\frac{\rho_{\tau}}{\gamma}\right)^{t}}{1-\frac{\rho_{\tau}}{\gamma}}
$$

therefore,

$$
\left\|\gamma^{-1} \sum_{i=0}^{t}\left(\frac{\rho_{\tau}}{\gamma}\right)^{i} \Phi^{T} \mathcal{L} \otimes B K \tilde{z}_{2}(t-i)\right\| \leq \frac{\sqrt{N} \lambda_{N}\|B K\|_{\infty}}{\left(\gamma-\rho_{\tau}\right)} \nu\left(1-\frac{\rho_{\tau}{ }^{t}}{\gamma}\right) u_{j}
$$

Note $\gamma \in\left(\rho_{\tau}, 1\right)$, using (34) - (36) therefore

$$
\left\|\tilde{\omega}_{2}(t+1)\right\| \leq \max \left\{\frac{\sqrt{N}\left(c_{\delta}+\lambda_{N}\|B K\| C_{x}\right)}{g(0) \gamma}, \frac{\sqrt{N} \lambda_{N}\|B K\|_{\infty}}{\left(\gamma-\rho_{\tau}\right)}\right\} \nu
$$


Thus, the quantizer is given as

$$
\begin{aligned}
\| & \left(I_{N} \otimes A+\mathcal{L} \otimes B K\right) z(t+1)-(\mathcal{L} \otimes B K) \omega(t+1) \|_{2} \\
\leq & \left(\left\|I_{N} \otimes A\right\|_{\infty}+\|\mathcal{L} \otimes B K\|_{\infty}\right)\|z(t+1)\|_{\infty}+\|\mathcal{L} \otimes B K\|_{\infty}\|\omega(t+1)\|_{2} \\
\leq & \frac{(1-\gamma)\left(\|A\|_{\infty}+2 d_{\max }\|B K\|_{\infty}\right)}{1+\gamma} u_{j}+\lambda_{N}\|B K\|_{\infty}\|\omega(t+1)\|_{2} \\
\leq & \left(\|A\|_{\infty}+2 d_{\max }\|B K\|_{\infty}\right) \nu u_{j}+\lambda_{N}\|B K\|_{\infty} \\
& \times \max \left\{\frac{\sqrt{N}\left(C_{\delta}+\lambda_{N}\|B K\| C_{x}\right)}{g(0) \gamma}, \frac{\sqrt{N} \lambda_{N}\|B K\|_{\infty}}{\left(\gamma-\rho_{\tau}\right)}\right\} \nu \\
= & M(\tau, \gamma)<\left\lfloor M(\tau, \gamma)-\frac{1}{2}\right\rfloor+\frac{3}{2} \\
= & K_{1}(\tau, \gamma)+\frac{1}{2} \leq K+\frac{1}{2}
\end{aligned}
$$

For an unsaturated quantizer, $K_{1} \geq K$ and $(2 Q+1)$ is applied to quantizer (6), likewise the quantizer is not saturated.

Taking $\|\omega(0)\|_{\infty} \leq \frac{\|\delta(0)\|_{\infty}}{g(0)}$, by (37) and (18) then

$$
\sup _{t \geq 0}\|\omega(t)\|_{\infty} \leq \max \left\{\frac{C_{\delta}}{g(0)}, \frac{\sqrt{N} \lambda_{N}\|B K\|_{\infty}}{\left(\gamma-\rho_{\tau}\right)}\right\}<\infty
$$

Thus, $\omega(t)$ and $0<\gamma<1$, signifies that $\lim _{t \rightarrow \infty}\|\delta(t)\|_{\infty}=0$. Hence, from (33)-(36) taking $\delta(t)=g(t+1) \omega(t)$ gives

$$
\begin{aligned}
\frac{r_{\text {asym }}}{\gamma} & =\sup _{\delta(0) \neq 0} \lim _{t \rightarrow \infty} \frac{\|\delta(t)\|_{2}}{\|\delta(0)\|_{2}} \\
& =\gamma\left(\rho_{\tau}\right)^{t}+\frac{\sqrt{N}\|B K\| C_{x} \lambda_{N}}{\|\delta(0)\|_{2} g(0)}\left(\rho_{\tau}\right)^{t}+\frac{\gamma g(0) \sqrt{N} \lambda_{N}\|B K\|_{\infty}}{\|\delta(0)\|_{2}\left(\gamma-\rho_{\tau}\right)} \nu \quad \forall \delta(0) \neq 0
\end{aligned}
$$

if $\rho_{\tau}<<\gamma,(41)$ becomes

$$
\begin{aligned}
& =\lim _{t \rightarrow \infty} \gamma^{-t}\|\delta(t)\|_{2} \\
& =\frac{g(0) \sqrt{N} \lambda_{N}\|B K\|_{\infty}}{\left(\gamma-\rho_{\tau}\right)} \nu
\end{aligned}
$$

Remark 3 Since $I_{N}$ and $\mathcal{L}$ are stochastic, likewise the updated matrix $P$, this induces the undirected graph as $\mathcal{L}$ but with different adjacency weights. Hence, the stochastic matrix $\mathcal{L}$ satisfies assumption $\mathbf{A} 1$, as well as $P$.

Remark 4 The performance of MASs is influenced; when the initial state is considerable large, the input signal tends to be saturated, causing a rapid decrease in $g(t)$. Thus, resulting in a period of overshoot, hence the immediate reduction in $g(t)$ stops saturation while the state decays exponentially. 
Remark 5 [15] The set of consensus conditions $\gamma \in\left(\rho_{\tau}, 1\right)$ with $\rho_{\tau}=\max _{2 \leq i \leq N} \mid A-$ $B K \lambda_{i} \mid$ where $\tau<K \lambda_{N}$ determines the convergence analysis of MASs, considering the decomposition matrix, eigenvalue spectrum of an undirected symmetric network $\mathcal{L}$ and the associated Laplacian matrix $\mathcal{L}$.

Remark 6 The connection between the scaling factor (42) and consensus error of the closed-loop system determines the convergence rate of the closed-loop system. Hence, in Theorem 1 the scaling factor with the quantizer decays exponentially. Thus, with (9) and (11) average consensus can be reached asymptotically. Note for any specified $\tau$ and $\gamma$; the number of bits is approximately conservative. Besides, the required number of bits, control gain $\tau$ and scaling factor $\gamma$ shows how the number $K_{1}(\tau, \gamma)$ are related. Likewise, based on the logarithmic quantizer parameter given in Theorem 1 as $\gamma$ reduces, consensus error converges to zero faster.

\section{Simulation Result}

Consider a dynamic undirected network systems of four agents such that the adjacent matrix $\mathcal{A}$ is $0-1$. This implies that $a_{i j}=1$, if $(i, j) \in \mathcal{E}$, otherwise, $a_{i j}=0$ shown in Figure 2 with the system matrices in (2) as

$$
x_{i}(t+1)=\left[\begin{array}{ll}
1 & 0 \\
1 & 1
\end{array}\right] x_{i}(t)+\left[\begin{array}{l}
1 \\
1
\end{array}\right] u_{i}(t), \quad i=1,2, . ., N
$$

Let $K=[0.4,0.2]$ and $\gamma \in(0.99967,1)$, while the initial states are chosen as $x_{i}(0)=i, \quad i=1,2, \ldots, N$ the consensus performance is shown in Figure 3.

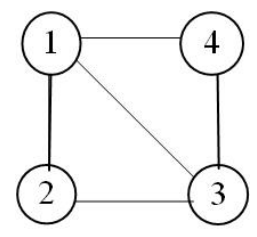

Fig. 2 Undirected Network Topology

\section{Conclusions}

The consensus of an undirected network of MASs has been studied under a finite quantized information network. The undirected network exchange information through the communication channel which has a couple of encoder and decoder that estimate and generates the neighbour state protocol for the system. Besides, the desired asymptotic convergence can be reached by rightly choosing the appropriate parameters of the logarithmic quantizer. For future research, the focus will be on observer-based consensusability using finite-level logarithmic-quantized feedback control. 


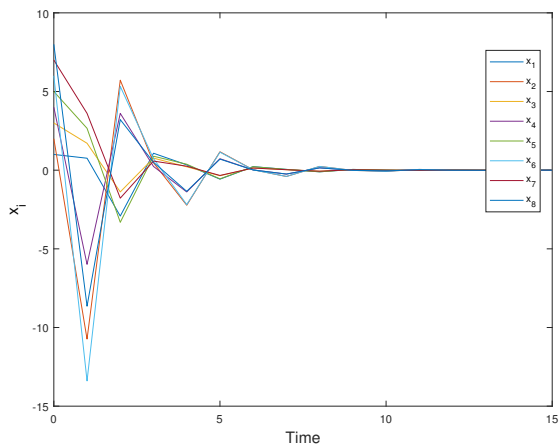

Fig. 3 Trajectories of State for Four Agents with Initial States $x_{i}(0)$

\section{Conflict of Interest, Funder and Data Availability Statement}

Conflict of Interest: The authors declare that they have no conflict of interest Funding: No funder/grant exist Data Availability Statement: Data sharing not applicable to this article as no datasets are generated are generate or analysed during the current study

\section{References}

1. Borkar, V., Varaiya, P. Asymptotic agreement in distributed estimation. IEEE Transactions on Automatic Control, 27(3), pp.650-655, (1982).

2. Brockett, R. W., Liberzon, D. Quantized feedback stabilization of linear systems. IEEE transactions on Automatic Control, 45(7), pp.1279-1289, (2000).

3. Carli, R., Fagnani, F., Frasca, P., Zampieri, S. Efficient quantization for average consensus. arXiv preprint arXiv:0903.1337, (2009).

4. Carli, R., Fagnani, F., Frasca, P., Zampieri, S. Gossip consensus algorithms via quantized communication. Automatica 46(1), pp.70-80, (2010).

5. Chatterjee, S., Seneta, E. Towards consensus: some convergence theorems on repeated averaging. Journal of Applied Probability, pp.89-97, (1977).

6. Fax, J.A., Murray, R.M. Information flow and cooperative control of vehicle formations. IEEE transactions on automatic control, 49(9), pp.1465-1476, (2004).

7. Fu, M. Quantization for feedback control and estimation. 27th Chinese Control Conference IEEE pp.751-756, (2008).

8. Fu, M., de Souza, C.E. State estimation for linear discrete-time systems using quantized measurements. Automatic 45(12), pp.2937-2945, (2009)

9. Fu, M., Xie, L. The sector bound approach to quantized feedback control. Transactions on Automatic control. IEEE.

textbf50(11), pp.1698-1711.

10. Fu, M., Xie, L. Finite-level quantized feedback control for linear systems. IEEE Transactions on Automatic Control, 54(5), pp.1165-1170, (2009).

11. Fu, M., Xie, L. Quantized feedback control for linear uncertain systems. International Journal of Robust and Nonlinear Control, 20(8), pp.843-857, (2010).

12. Gilardoni, G. L., Clayton, M.K., On reaching a consensus using DeGroot's iterative pooling. The Annals of Statistics, pp.391-401, (1993).

13. Jadbabaie, A., Lin, J., Morse, A.S. Coordination of groups of mobile autonomous agents using nearest neighbor rules. IEEE Transactions on automatic control. 48(6), pp.988-1001, (2003). 
14. Li, Z., Duan, Z., Chen, G., Huang, L., Consensus of multiagent systems and synchronization of complex networks: a unified viewpoint. IEEE Transactions on Circuits and Systems I: Regular Papers, 57(1), pp.213-224, (2010).

15. Li, T., Fu, M., Xie, L. Zhang, J.F. Distributed consensus with limited communication data rate. IEEE Transactions on Automatic Control, 56(2), pp.279-292, (2011).

16. Liang, H., Zhang, Y., Huang, T., Ma, H. Prescribed performance cooperative control for multiagent systems with input quantization. IEEE Transactions on cybernetics, 50(5), pp. 1810-1819, (2019).

17. Lin, Z., Broucke, M., Francis, B. Local control strategies for groups of mobile autonomous agents. IEEE Transactions on automatic control, 49(4), pp.622-629, (2004).

18. Lin, P., Jia, Y. Consensus of second-order discrete-time multi-agent systems with nonuniform time-delays and dynamically changing topologies. Automatica, 45(9), pp.2154-2158, (2009).

19. Liu, C., Zhou, Q., Liu, Y. (2014). Consensus of Single-Integrator Multi-Agent Systems at a Preset Time. International Conference in Swarm Intelligence. pp. 431-441 Springer International Publishing.

20. Moreau, L. Stability of multiagent systems with time-dependent communication links. IEEE Transactions on automatic control, 50(2), pp.169-182, (2005).

21. Olfati-Saber, R., Murray, R.M. . Consensus problems in networks of agents with switching topology and time-delays. IEEE Transactions on automatic control, 49(9), pp.15201533, (2004)

22. Nuga, O.O., Li, Z. Ding, Z., Quantized consensus of nonlinear systems. In 2019 IEEE 15th International Conference on Control and Automation (ICCA) (pp. 1032-1037), (2019).

23. Qiu, Z., Hong, Y., Xie, L. Quantized leaderless and leader-following consensus of highorder multi-agent systems with limited data rate. In 52nd IEEE Conference on Decision and Control, (pp. 6759-6764), (2013).

24. Ren, W. Multi-vehicle consensus with a time-varying reference state. Systems and Control Letters, 56(7), pp.474-483, (2007), (2013).

25. Ren, W., Beard, R.W. Consensus seeking in multiagent systems under dynamically changing interaction topologies. IEEE Transactions on automatic control, 50(5), pp.655$661,(2005)$.

26. Tsitsiklis, J. N., Bertsekas, D.P., Athans, M. Distributed asynchronous deterministic and stochastic gradient optimization algorithms. American Control Conference, pp. 484489, (1984).

27. Wen, G., Duan, Z., Ren, W., Chen, G. Distributed consensus of multi-agent systems with general linear node dynamics and intermittent communications. International Journal of Robust and Nonlinear Control, 24(16), 2438-2457, (2014).

28. Xie, G., Wang, L.. Consensus control for a class of networks of dynamic agents. International Journal of Robust and Nonlinear Control, 171011, pp.941-959, (2007).

29. You, K. and Xie, L. Network topology and communication data rate for consensusability of discrete-time multi-agent systems. IEEE Transactions on Automatic Control, 56(10), pp.2262-2275, (2011).

30. Zhang, Z., Zhang, L., Hao, F., Wang, L. Leader-following consensus for linear and Lipschitz nonlinear multiagent systems with quantized communication. IEEE transactions on cybernetics, $\mathbf{4 7}(8)$, pp. 1970-1982, (2016).

31. Zhenxing, L., Haibo, J. Quantized consensus for linear discrete-time multi-agent systems. In Control Automation Robotics and Vision (ICARCV), 2012 12th International Conference pp. 1472-1476 (2012). 